\title{
Non-Fragile Sliding Mode Control of Uncertain Chaotic Systems
}

\author{
Leipo Liu, ${ }^{1}$ Zhengzhi Han, ${ }^{2}$ and Zhumu Fu' \\ ${ }^{1}$ College of Electrical and Information Engineering, Henan University of Science and Technology, Luoyang 471003, China \\ ${ }^{2}$ School of Electrical and Information Engineering, Shanghai Jiao Tong University, Shanghai 200240, China
}

Correspondence should be addressed to Leipo Liu, liuleipo123@yahoo.com.cn

Received 5 April 2011; Accepted 27 June 2011

Academic Editor: Onur Toker

Copyright ( 2011 Leipo Liu et al. This is an open access article distributed under the Creative Commons Attribution License, which permits unrestricted use, distribution, and reproduction in any medium, provided the original work is properly cited.

\begin{abstract}
This paper is concerned with non-fragile sliding mode control of uncertain chaotic systems with external disturbance. Firstly, a new sliding surface is proposed, and sufficient conditions are derived to guarantee that sliding mode dynamics is asymptotically stable with a generalized $\mathrm{H}_{2}$ disturbance rejection level. Secondly, non-fragile sliding mode controller is established to make the state of system reach the sliding surface in a finite time. Finally, an example is given to illustrate the effectiveness of the proposed method.
\end{abstract}

\section{Introduction}

Chaotic behavior is a seemingly random phenomenon of a deterministic system that is characterized by sensitive deaspendence on initial conditions. Many electronic, mechanical, and chemical systems exhibit chaotic dynamics. Therefore, chaos is a very interesting nonlinear phenomenon, and control of chaotic systems has been paid much attention by researchers since the pioneering work of Ott et al. [1]. The main aim of chaos control is to suppress chaotic behavior and to stabilize the chaotic system, and various effective techniques have been presented and successfully applied to obtain chaos control such as adaptive control [2], backstepping control [3], fuzzy control [4], optimal control [5], and sliding mode control [6-9].

Among the above-mentioned methods, sliding mode control is a very effective approach to control chaos because of its attractive features such as fast response, good transient response, and insensitivity to variations in system parameters and external disturbances [6-11]. Furthermore, in some engineering practices, for example, in order to avoid actuator saturation, one often guarantees that whenever the exogenous input has bounded energy, the controlled output should have a specified maximum peak value. In this case, generalized $\mathrm{H}_{2}$ control is a good tool $[12,13]$.

Generally speaking, accurate controllers are required to stabilize control systems, so all of the controller coefficients are exact values in designing a desired controller. However, in practice, the uncertainty is not avoided, and it may be caused by many reasons, such as finite word length in digital systems, the imprecision inherent in analog systems, and the need for additional tuning of parameters in the final controller implementation [14]. The existence of uncertainty leads to the study of non-fragile control problem, that is, to design a controller such that the controller is insensitive to uncertainties. Many results about non-fragile control problems have been reported [15-17]. However, so far, nonfragile sliding mode control problem for uncertain chaotic systems has been paid little attention.

In this paper, the problem of non-fragile sliding mode control of uncertain chaotic systems with external disturbance is considered. Firstly, a new integral-type sliding surface is proposed, and the reaching phase is eliminated, and the proposed sliding surface function includes a normbounded parameter uncertainty; this term is added not only to make the design of sliding surface more relaxed, but also to guarantee that the sliding mode dynamics has better robust performance. For both the cases with additive and multiplicative uncertainties, sufficient conditions are derived to make sliding mode dynamics stable with a generalized $\mathrm{H}_{2}$ performance. Secondly, non-fragile sliding mode controller is established to make the state of system reach the sliding surface in a finite time. Finally, an example is given to illustrate the effectiveness of the proposed method.

Notations. The following notations will be used throughout this paper. $R^{n}$ and $R^{n \times m}$ denote, respectively, the $n$-dimensional Euclidean space and the space of $n \times m$ real matrices. 


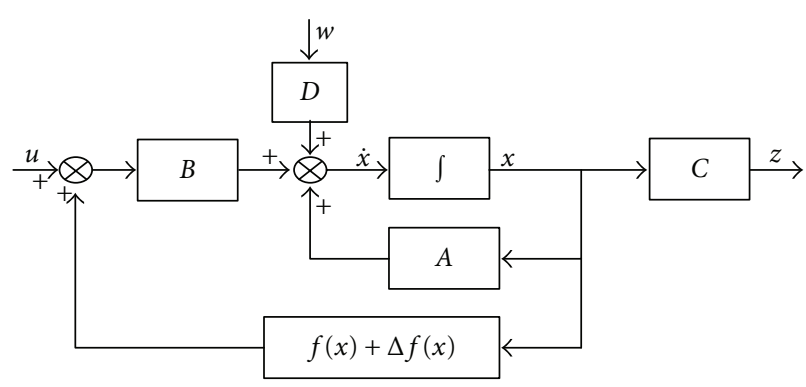

Figure 1: The block diagram of the system (1).

For a real symmetric matrix $M, M>0$ means $M$ is positive definite. $L_{2}[0, \infty)$ is the space of square-integrable vector functions over $[0, \infty)$, and $L_{\infty}$ is the space of essential bounded functions. $*$ represents the symmetric form of matrix.

\section{Problem Formulation}

Consider the following chaotic system:

$$
\begin{gathered}
\dot{x}(t)=A x+B(f(x)+\Delta f(x)+u(t))+D w(t), \\
z(t)=C x(t),
\end{gathered}
$$

where $x(t) \in R^{n}$ is the state vector, $u(t) \in R^{m}$ is the control input. $z(t) \in R^{q}$ is the system output. $f(x)$ is a nonlinear real-valued function. $A, B, C$, and $D$ are matrices with compatible dimensions and $(A, B)$ is completely controllable. $B$ is of full column rank. $w(t)$ is a bounded disturbance. $\Delta f(x)$ represents the uncertainty part, satisfying $\|\Delta f(x)\| \leq \rho$ with a positive constant $\rho$.

The block diagram of the system (1) is given in Figure 1.

The following lemmas are necessary for future discussion.

Lemma 1 (see [8]). For a given matrix $S=\left[\begin{array}{ll}S_{11} & S_{12} \\ S_{12}^{T} & S_{22}\end{array}\right]$ with $S_{11}=$ $S_{11}^{T}, S_{22}=S_{22}^{T}$, then the following conditions are equivalent:

(1) $S<0$,

(2) $S_{22}<0, S_{11}-S_{12} S_{22}^{-1} S_{12}^{T}<0$,

(3) $S_{11}<0, S_{22}-S_{12}^{T} S_{11}^{-1} S_{12}<0$.

Lemma 2 (see [17]). Let $\Omega, \Gamma$ and $\Xi$ be matrices of appropriate dimensions, and let $\Omega$ be symmetrical. Then

$$
\Omega+\Gamma F(t) \Xi+(\Gamma F(t) \Xi)^{T}<0,
$$

for an uncertain matrix $F(t)$ satisfying $F^{T}(t) F(t) \leq I$ if and only if there exists a scalar $\varepsilon>0$ such that

$$
\Omega+\varepsilon \Gamma \Gamma^{T}+\frac{1}{\varepsilon}\left(\Xi^{T} \Xi\right)<0 .
$$

\section{Main Results}

For system (1), firstly, a new sliding surface is designed, and sufficient conditions are given to guarantee that the sliding mode dynamics is asymptotically stable with a generalized $\mathrm{H}_{2}$-norm bound $\gamma$. Secondly, non-fragile sliding mode controller is designed to drive the state arriving at the sliding surface in a finite time.

3.1. Sliding Surface Design. For system (1), we design the following integral-type sliding surface function:

$$
s(t)=G\left[x(t)-x(0)-\int_{0}^{t}(A+B(K+\Delta K(s))) x(s) d s\right],
$$

where $G$ is a matrix such that $G B$ is invertible. $K>0$ is a gain matrix to be chosen later, and the matrix $\Delta K(t)$ represents the norm-bounded parameter uncertainty.

In this paper, the following two classes of parameter uncertainty will be considered:

(a) $\Delta K(t)$ is with the norm-bounded additive form

$$
\Delta K(t)=\Delta_{1}=H_{1} F_{1}(t) E_{1},
$$

where $H_{1}$ and $E_{1}$ are known matrices and $F_{1}(t)$ is an unknown matrix satisfying

$$
F_{1}^{T}(t) F_{1}(t) \leq I
$$

(b) $\Delta K(t)$ is with the norm-bounded multiplicative form

$$
\Delta K(t)=\Delta_{2}=H_{2} F_{2}(t) E_{2} K,
$$

where $H_{2}$ and $E_{2}$ are known matrices, and $F_{2}(t)$ is an unknown matrix satisfying

$$
F_{2}^{T}(t) F_{2}(t) \leq I .
$$

Remark 3. From (4), we have $s(0)=0$. It is worth to note that the integral-type sliding surface function (4) is different from the existing methods, for example, those presented in [10, 11 ], since it takes the term $\Delta K(t)$ into account. Moreover, these existing results can be considered as a special form of the integral-type sliding surface designed in this paper, when $\Delta K(t)$ is zero. In fact, uncertainty $\Delta K(t)$ is added not only to make the design of sliding surface more relaxed, but also to guarantee that the sliding mode dynamics has better robust performance.

Let $\dot{s}(t)=0$. Then, the equivalent control law can be obtained as follows:

$u_{\mathrm{eq}}(t)=(K+\Delta K(t)) x(t)-(G B)^{-1} G D w(t)-f(x)-\Delta f(x)$.

Substituting (9) into the system (1) and denoting $\bar{D}=$ $D-B(G B)^{-1} G D$, sliding mode dynamics and the output equation can be formulated as

$$
\begin{gathered}
\dot{x}(t)=(A+B K+B \Delta K(t)) x(t)+\bar{D} w(t), \\
z(t)=C x(t) .
\end{gathered}
$$


Definition 4. Sliding mode dynamics (10) is asymptotically stable with a generalized $\mathrm{H}_{2}$-norm bound $\gamma$ if the following conditions hold.

(i) When $w(t)=0$, sliding mode dynamics (10) is asymptotically stable.

(ii) Sliding mode dynamics (10) has a given generalized $H_{2}$ (or $L_{2}-L_{\infty}$ ) disturbance rejection level; that is, the $L_{2}-L_{\infty}$ gain from disturbance input $w(t)$ to control output $z(t)$ of the system (10) is less than a given value. It is to make, under the zero-valued initial condition, the following inequality hold:

$$
\sup _{w \in L_{2}-0} \frac{\|z\|_{\infty}^{2}}{\|w\|_{2}^{2}}<\gamma^{2}
$$

where

$$
\|w\|_{2}^{2}=\int_{0}^{\infty} w^{T}(t) w(t) d t, \quad\|z\|_{\infty}^{2}=\sup _{t}\left\{z^{T}(t) z(t)\right\} .
$$

Theorem 5. Consider the system (10) and a given constant $\gamma>$ 0 . If there exist matrices $P>0$ and $K$ such that the following matrix inequalities are true

$$
\begin{aligned}
\Theta & =\left[\begin{array}{cc}
P(A+B K+B \Delta K(t))+(A+B K+B \Delta K(t))^{T} P & P \bar{D} \\
* & -I
\end{array}\right] \\
<0 & \\
& {\left[\begin{array}{cc}
P & C^{T} \\
* & \gamma^{2} I
\end{array}\right]>0 }
\end{aligned}
$$

then the sliding mode dynamics (10) is asymptotically stable with a generalized $\mathrm{H}_{2}$-norm bound $\gamma$.

Proof. Consider the following Lyapunov functional candidate:

$$
V_{1}(t)=x^{T}(t) P x(t) .
$$

The time derivative of $V_{1}(t)$ along the trajectories of system (10) with $w(t)=0$ is given by

$$
\begin{aligned}
\dot{V}_{1}(t)=x^{T}(t)[ & P(A+B K+B \Delta K(t)) \\
& \left.+(A+B K+B \Delta K(t))^{T} P\right] x(t) .
\end{aligned}
$$

From (13), $\Theta<0$ implies $P(A+B K+B \Delta K(t))+(A+$ $B K+B \Delta K(t))^{T} P<0$, then we have

$$
\dot{V}_{1}(t)<0,
$$

which implies sliding mode dynamics (10) is asymptotically stable, when $w(t)=0$.

In order to construct the generalized $H_{2}\left(L_{2}-L_{\infty}\right)$ performance index, we assume the zero-valued initial condition, then $\left.V_{1}(t)\right|_{t=0}=0$. Now, we consider the following cost performance:

$$
J=V_{1}(t)-\int_{0}^{t} w^{T}(s) w(s) d s .
$$

For any nonzero $w(t) \in L_{2}[0, \infty)$, we have

$$
\begin{aligned}
J & =\int_{0}^{t}\left[\dot{V}_{1}(s)-w^{T}(s) w(s)\right] d s \\
& =\int_{0}^{t} \xi^{T} \Theta \xi d s,
\end{aligned}
$$

where $\xi=\left[x^{T}(s) w^{T}(s)\right]^{T}$.

From (13), we obtain

$$
J=V_{1}(t)-\int_{0}^{t} w^{T}(s) w(s) d s<0 .
$$

Equation (20) leads to

$$
x^{T}(t) P x(t)=V_{1}(t)<\int_{0}^{t} w^{T}(s) w(s) d s .
$$

For (14), by Lemma 1, we have

$$
C^{T} C<\gamma^{2} P
$$

which leads to

$$
\begin{aligned}
z^{T}(t) z(t) & =x^{T}(t) C^{T} C x(t) \\
& <\gamma^{2} x^{T}(t) P x(t) \\
& <\gamma^{2} \int_{0}^{t} w^{T}(s) w(s) d s \\
& <\gamma^{2} \int_{0}^{\infty} w^{T}(s) w(s) d s .
\end{aligned}
$$

For any time $t \geq 0$, taking the maximum value of $z^{T}(t) z(t)$, we have

$$
\|z\|_{\infty}^{2}<\gamma^{2}\|w(t)\|_{2}^{2} .
$$

The proof is completed.

For sliding mode dynamics (10) with additive and multiplicative uncertainties, the following theorems give some sufficient conditions which guarantee the sliding mode dynamics is asymptotically stable with a generalized $\mathrm{H}_{2}$ norm bound $\gamma$.

Theorem 6. Consider the system (10), uncertainty $\Delta K(t)$ in (5), and a given constant $\gamma>0$. If there exist matrices $X>0$, $Y$ and a scalar $\varepsilon_{1}>0$ such that the following linear matrix inequalities (LMIs) are true

$$
\left[\begin{array}{ccc}
A X+X A^{T}+B Y+Y^{T} B^{T}+\varepsilon_{1} B H_{1} H_{1}^{T} B^{T} & \bar{D} & X E_{1}^{T} \\
* & -I & 0 \\
* & * & -\varepsilon_{1} I
\end{array}\right]<0
$$

$$
\left[\begin{array}{cc}
X & X C^{T} \\
* & \gamma^{2} I
\end{array}\right]>0
$$


then the sliding mode dynamics (10) is asymptotically stable with a generalized $\mathrm{H}_{2}$-norm bound $\gamma$. Furthermore, the gain matrix $K$ is chosen as $K=Y X^{-1}$.

Proof. Pre- and postmultiplying (13) and (14) by $\operatorname{diag}\{X, I\}$ with $X=P^{-1}$, respectively, we have

$$
\left[\begin{array}{cc}
(A+B K+B \Delta K(t)) X+X(A+B K+B \Delta K(t))^{T} & \bar{D} \\
* & -I
\end{array}\right]<0,
$$

and (26).

From (27), we can obtain

$$
\begin{gathered}
{\left[\begin{array}{cc}
(A+B K) X+X(A+B K)^{T} & \bar{D} \\
* & -I
\end{array}\right]+\left[\begin{array}{c}
B H_{1} \\
0
\end{array}\right] F_{1}(t)\left[\begin{array}{lll}
E_{1} & X & 0
\end{array}\right]} \\
+\left(\left[\begin{array}{c}
B H_{1} \\
0
\end{array}\right] F_{1}(t)\left[\begin{array}{lll}
E_{1} & X & 0
\end{array}\right]\right)^{T}<0 .
\end{gathered}
$$

From Lemma 2, (28) holds if and only if there exists a scalar $\varepsilon_{1}>0$ such that the following inequality holds:

$$
\begin{aligned}
& {\left[\begin{array}{cc}
(A+B K) X+X(A+B K)^{T} & \bar{D} \\
* & -I
\end{array}\right]+\varepsilon_{1}\left[\begin{array}{c}
B H_{1} \\
0
\end{array}\right]\left[\begin{array}{c}
B H_{1} \\
0
\end{array}\right]^{T}} \\
& +\frac{1}{\varepsilon_{1}}\left[\begin{array}{c}
X E_{1}^{T} \\
0
\end{array}\right]\left[\begin{array}{lll}
E_{1} & X & 0
\end{array}\right]<0,
\end{aligned}
$$

which, according to Lemma 1, is equivalent to (25).

The proof is completed.

Theorem 7. Consider the system (10), uncertainty $\Delta K(t)$ in (7), and a given constant $\gamma>0$. If there exist matrices $X>0$, $Y$ and the scalar $\varepsilon_{2}>0$ such that the following linear matrix inequalities (LMIs) are true

$$
\left[\begin{array}{ccc}
A X+X A^{T}+B Y+Y^{T} B^{T}+\varepsilon_{2} B H_{2} H_{2}^{T} B^{T} & \bar{D} & Y E_{2}^{T} \\
* & -I & 0 \\
* & * & -\varepsilon_{2} I
\end{array}\right]<0,
$$

$$
\left[\begin{array}{cc}
X & X C^{T} \\
* & \gamma^{2} I
\end{array}\right]>0
$$

then the sliding mode dynamics (10) is asymptotically stable with a generalized $\mathrm{H}_{2}$-norm bound $\gamma$. Furthermore, the gain matrix $K$ is chosen as $K=Y X^{-1}$.

Proof. The proof is similar to Theorem 6, and omitted.

3.2. Sliding Mode Controller Design. The input $u(t)$ in system (1) should be appropriately designed such that the state $x(t)$ in system (1) reaches the sliding surface $s(t)=0$ within a finite time. The non-fragile sliding mode controller $u(t)$ is as follows:

$$
\begin{aligned}
u(t)= & (K+\Delta K(t)) x(t)-f(x)-(G B)^{-1} \\
& \times[\alpha s(t)+(\|G D\| \cdot\|w(t)\|+\|G B\| \cdot \rho+\beta) \operatorname{sgn}(s(t))],
\end{aligned}
$$

where $\operatorname{sgn}(s(t))=\left[\operatorname{sign}\left(s_{1}\right), \operatorname{sign}\left(s_{2}\right), \ldots, \operatorname{sign}\left(s_{m}\right)\right]^{T}, \alpha>0$ and $\beta>0$.

Theorem 8. Consider the system (1). If the input $u(t)$ is taken as (32), then the trajectory of the system (1) converges to the sliding surface $s(t)=0$ in a finite time.

Proof. Consider a Lyapunov function candidate as follows:

$$
V_{2}(t)=\frac{1}{2} s^{T}(t) s(t)
$$

Calculating the time derivative of $V_{2}(t)$ along the trajectory of system (1), we have

$$
\begin{aligned}
\dot{V}_{2}(t)=s^{T}(t)[ & -G B(K+\Delta K(t)) x(t)+G D w(t) \\
& +G B(u(t)+f(x)+\Delta f(x))] .
\end{aligned}
$$

Substituting (32) into (34) yields

$$
\begin{aligned}
& \dot{V}_{2}(t)=s^{T}(t)[-G B(K+\Delta K(t)) x(t)+G D w(t)+G B \Delta f(x) \\
&+G B f(x)+G B(K+\Delta K(t)) x(t) \\
&-G B f(x)-\alpha s(t) \\
&-(\|G D\| \cdot\|w(t)\|+\|G B\| \cdot \rho+\beta) \operatorname{sgn}(s(t))] \\
& \leq\|s(t)\| \cdot(\|G D\| \cdot\|w(t)\|+\|G B\| \cdot \rho)-\alpha\|s(t)\|^{2} \\
&-(\|G D\| \cdot\|w(t)\|+\|G B\| \cdot \rho+\beta)\|s(t)\| .
\end{aligned}
$$

Then, we have

$$
\dot{V}_{2}(t) \leq-\alpha\|s(t)\|^{2}-\beta\|s(t)\| .
$$

According to sliding mode theory, we conclude that the trajectory of the system (1) converges to the sliding surface $s(t)=0$ in a finite time and remains in it thereafter. Thus, the proof is completed.

\section{Simulation}

In this section, we use Genesio's chaotic system to verify the effectiveness of the method. Genesio's system with additional input is as follows:

$$
\begin{gathered}
\dot{x}(t)=A x+B(f(x)+\Delta f(x)+u(t))+D w(t), \\
z(t)=C x(t),
\end{gathered}
$$




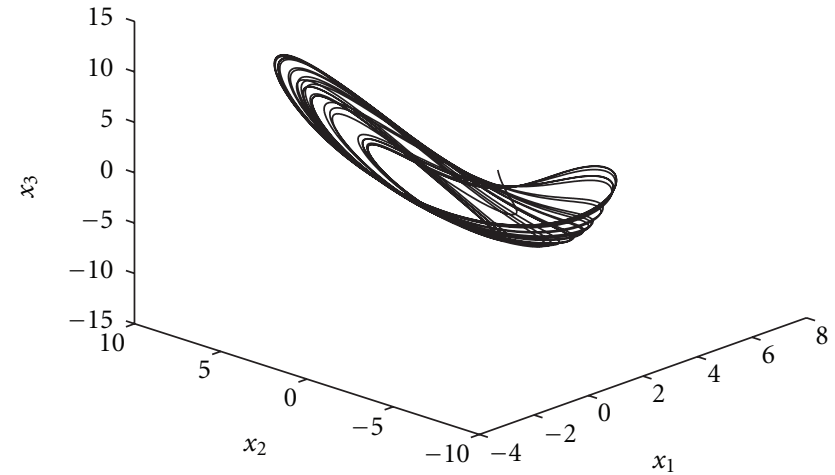

Figure 2: The phase curves of uncontrolled Genesio's system.

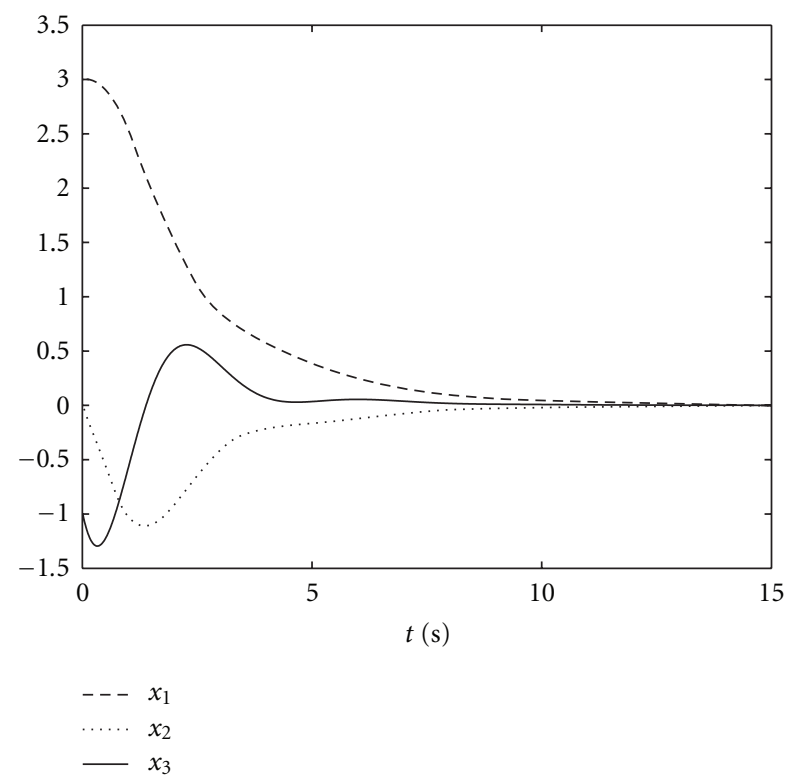

Figure 3: State $x(t)$ of the controlled Genesio's system.

where

$$
\begin{gathered}
A=\left[\begin{array}{ccc}
0 & 1 & 0 \\
0 & 0 & 1 \\
-6 & -2.92 & -1.2
\end{array}\right], \quad B=\left[\begin{array}{l}
0 \\
0 \\
1
\end{array}\right], \quad D=\left[\begin{array}{l}
1 \\
1 \\
1
\end{array}\right], \\
C=\left[\begin{array}{lll}
0 & 0 & -0.1
\end{array}\right], \quad \Delta f(x)=\sin \left(x_{3}(t)\right), \quad w(t)=\frac{e^{-t}}{10+t} .
\end{gathered}
$$

Without loss of generality, we consider $\Delta K(t)$ is with the norm-bounded additive form, where $H_{1}=\left[\begin{array}{lll}0 & 1 & 0\end{array}\right]$, $E_{1}=-0.1$ and $F_{1}(t)=0.1 \sin t, F_{1}(t)$ satisfies $F_{1}^{T}(t) F_{1}(t)=$ $0.01 \sin ^{2} t<1$. Furthermore, we choose $G=\left[\begin{array}{lll}0 & 0 & 1\end{array}\right]$ and $\gamma=0.3162$.

Then, solving LMIs (25) and (26) yields

$$
K=\left[\begin{array}{lll}
4.6545 & -0.8134 & -0.8913
\end{array}\right] .
$$

The initial value is $x(0)=\left[\begin{array}{lll}3 & 0 & -1\end{array}\right]^{T}$. The simulation results are shown in Figures 2, 3, 4, and 5 .

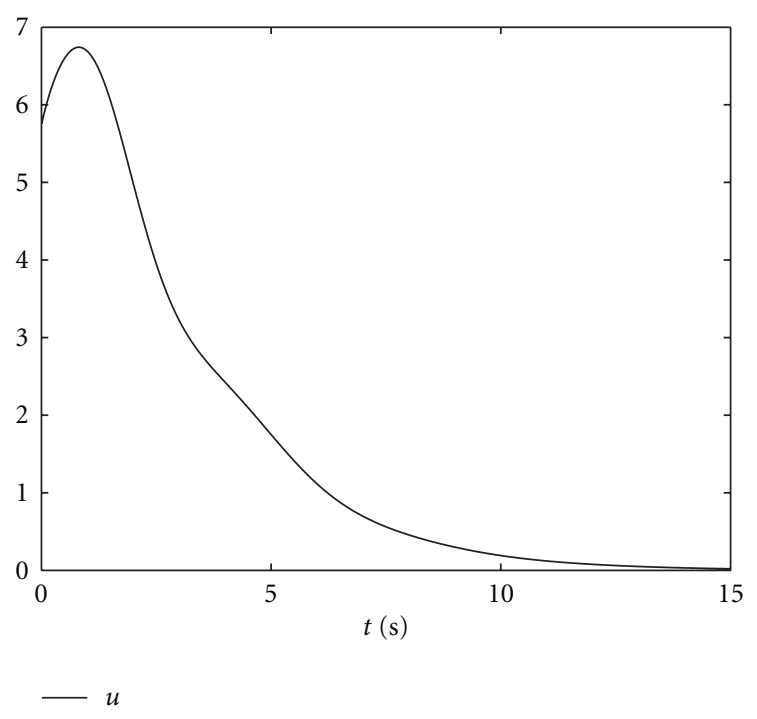

FIgURE 4: The control input $u(t)$.

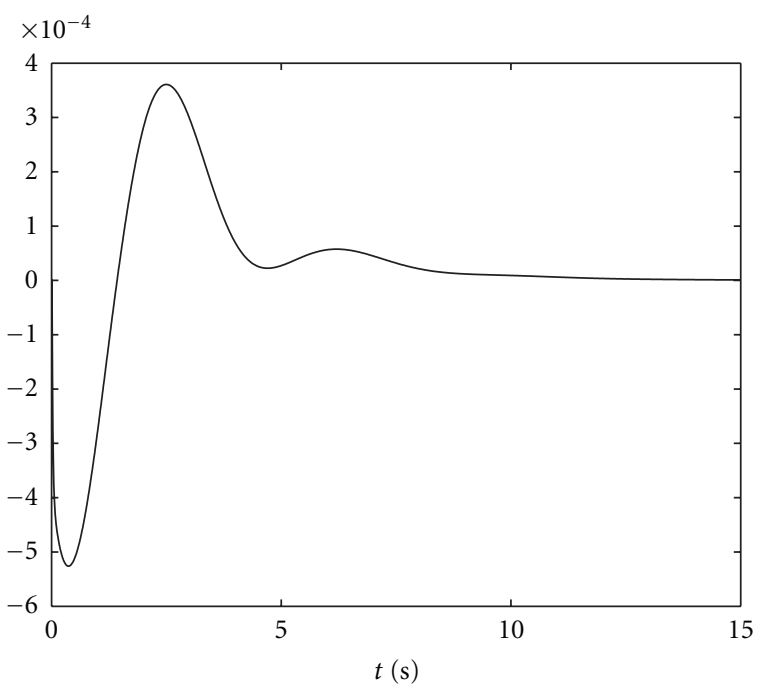

FIGURE 5: Sliding mode $s(t)$.

Figure 2 shows the phase curves of uncontrolled Genesio's system. Figure 3 shows the time response of the state $x(t)$ of the controlled Genesio's system. Figure 4 shows the control input response curve. Sliding mode response curve is shown in Figure 5. From the simulation results, it is concluded that the proposed method is effective.

\section{Conclusion}

In this paper, the problem of non-fragile sliding mode control of uncertain chaotic systems with external disturbance is investigated. A new sliding surface is proposed, and sufficient conditions are derived for asymptotic stability with a generalized $\mathrm{H}_{2}$ disturbance rejection level of sliding mode dynamics. Non-fragile sliding mode controller is established 
to make the state of system reach the sliding surface in a finite time. Finally, the simulation shows the effectiveness of the proposed method.

\section{Acknowledgment}

The authors are grateful for the support of the National Natural Science Foundation of China under Grants nos. 61074003 and 60904023.

\section{References}

[1] E. Ott, C. Grebogi, and J. A. Yorke, "Controlling chaos," Physical Review Letters, vol. 64, no. 11, pp. 1196-1199, 1990.

[2] C. Hua and X. Guan, "Adaptive control for chaotic systems," Chaos, Solitons and Fractals, vol. 22, no. 1, pp. 55-60, 2004.

[3] G. H. Li, "Projective synchronization of chaotic system using backstepping control," Chaos, Solitons and Fractals, vol. 29, no. 2, pp. 490-494, 2006.

[4] J. Wang, G. D. Qiao, and B. Deng, "Observer-based robust adaptive variable universe fuzzy control for chaotic system," Chaos, Solitons and Fractals, vol. 23, no. 3, pp. 1013-1032, 2005.

[5] C. Piccardi and L. L. Ghezzi, "Optimal control of a chaotic map: fixed point stabilization and attractor confinement," International Journal of Bifurcation and Chaos, vol. 7, no. 2, pp. 437-446, 1997.

[6] J. M. Nazzal and A. N. Natsheh, "Chaos control using slidingmode theory," Chaos, Solitons and Fractals, vol. 33, no. 2, pp. 695-702, 2007.

[7] L. Liu, Z. Han, and W. Li, "Global sliding mode control and application in chaotic systems," Nonlinear Dynamics, vol. 56, no. 1-2, pp. 193-198, 2009.

[8] L. Liu, Z. Han, and W. Li, " $H_{\infty}$ non-fragile observer-based sliding mode control for uncertain time-delay systems," Journal of the Franklin Institute, vol. 347, no. 2, pp. 567-576, 2010.

[9] L. Liu, Z. Han, and X. Cai, "Sliding mode control for polytopic differential inclusion systems," Mathematics and Computers in Simulation, vol. 79, no. 10, pp. 3018-3025, 2009.

[10] W. J. Cao and J. X. Xu, "Nonlinear integral-type sliding surface for both matched and unmatched uncertain systems," IEEE Transactions on Automatic Control, vol. 49, no. 8, pp. 1355-1360, 2004.

[11] F. Castaños and L. Fridman, "Analysis and design of integral sliding manifolds for systems with unmatched perturbations," IEEE Transactions on Automatic Control, vol. 51, no. 5, pp. 853-858, 2006.

[12] D. Du, S. Zhou, and B. Zhang, "Generalized $H_{2}$ output feedback controller design for uncertain discrete-time switched systems via switched Lyapunov functions," Nonlinear Analysis, Theory, Methods and Applications, vol. 65, no. 11, pp. 2135-2146, 2006.

[13] F. Liu, "Robust generalized $\mathrm{H}_{2}$ control: time-delay case," Journal of Systems Engineering and Electronics, vol. 15, no. 4, pp. 605-609, 2004.

[14] M. Gevers and G. Li, Parameterizations on Control, Estimations and Filtering Problem: Accuracy Aspects, Springer, Berlin, Germany, 1993.

[15] J. H. Kim and D. C. Oh, "Robust and non-fragile $H_{\infty}$ control for descriptor systems with parameter uncertainties and time delay," International Journal of Control, Automation and Systems, vol. 5, no. 1, pp. 8-14, 2007.
[16] Y. Y. Hou, T. L. Liao, J. J. Yan, and C. H. Lien, "Non-fragile $H_{\infty}$ control for singular systems with state and input timevarying delays," International Journal of Nonlinear Sciences and Numerical Simulation, vol. 8, no. 1, pp. 31-40, 2007.

[17] S. Wo, Y. Zou, Q. Chen, and S. Xu, "Non-fragile controller design for discrete descriptor systems," Journal of the Franklin Institute, vol. 346, no. 9, pp. 914-922, 2009. 

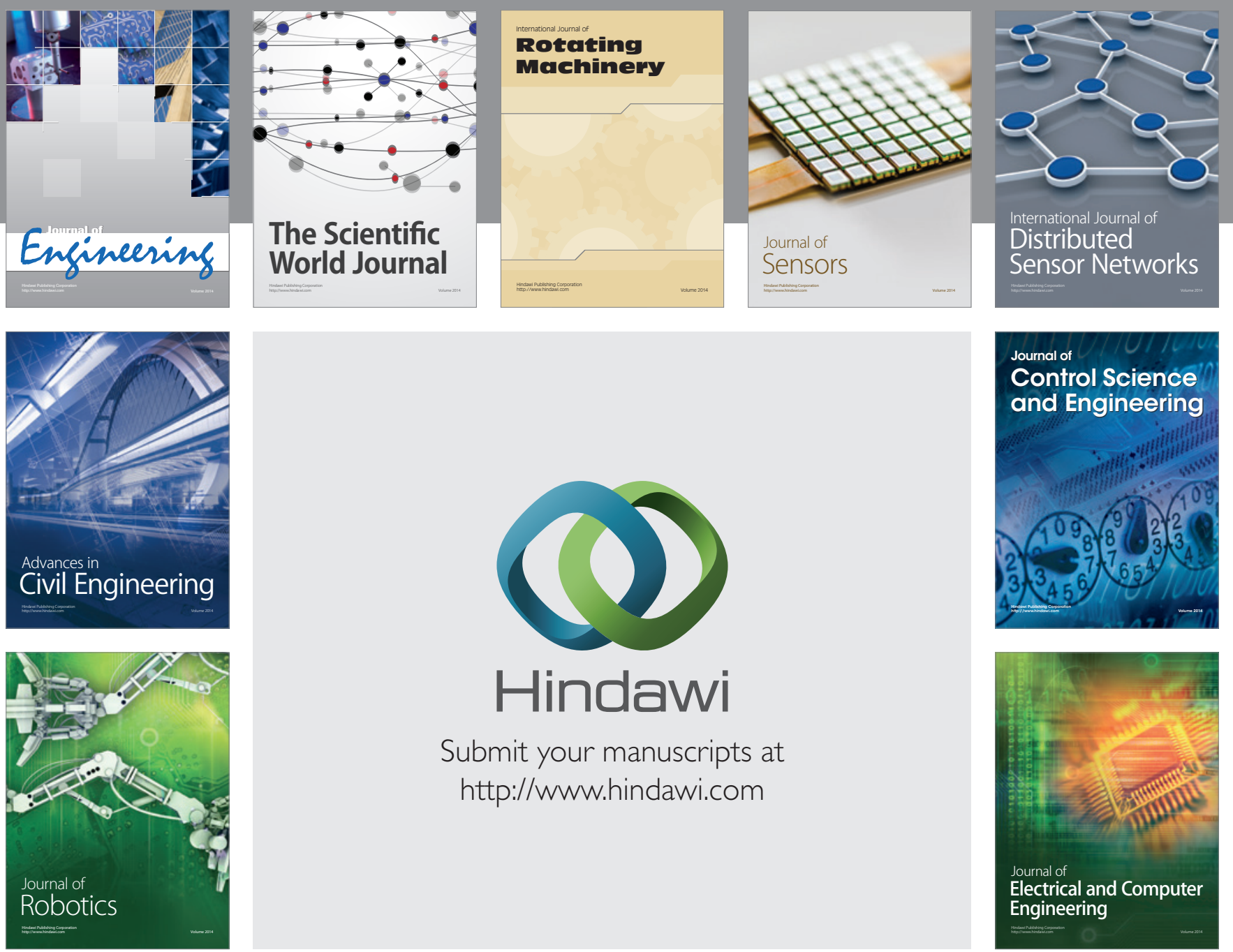

Submit your manuscripts at

http://www.hindawi.com
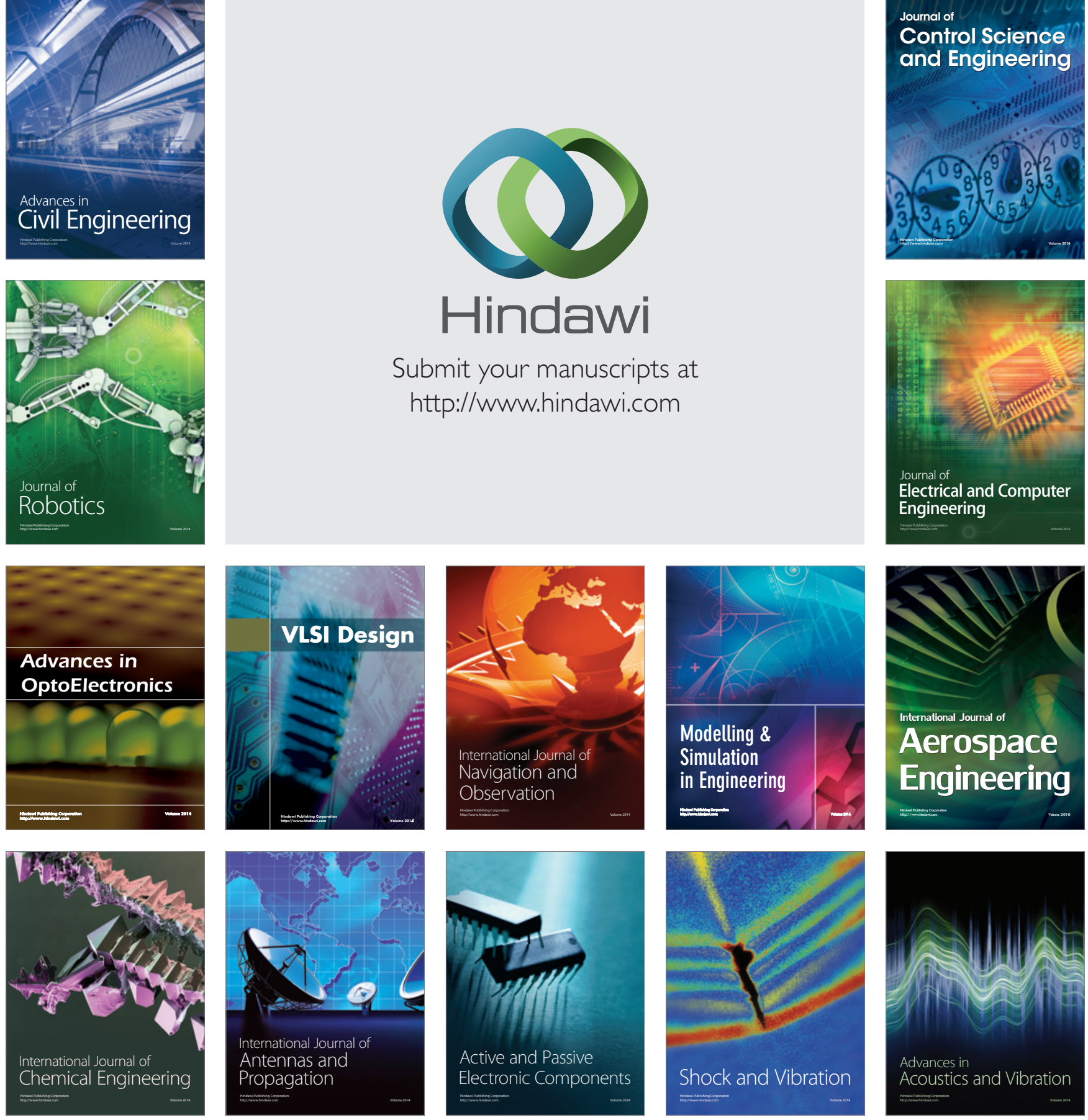